\title{
Fourier-Series Representation of Discontinuous Functions and Its Physical Applications
}

\author{
Sami M. Al-Jaber, Iyad Saadeddin* \\ Department of Physics, An-Najah National University, Nablus, Palestine \\ Email: Jaber@najah.edu, ‘iyads@najah.edu
}

How to cite this paper: Al-Jaber, S.M. and Saadeddin, I. (2019) Fourier-Series Representation of Discontinuous Functions and Its Physical Applications. Applied Mathematics, 10, 226-233. https://doi.org/10.4236/am.2019.104017

Received: March 25, 2019

Accepted: April 22, 2019

Published: April 25, 2019

Copyright $\odot 2019$ by author(s) and Scientific Research Publishing Inc. This work is licensed under the Creative Commons Attribution International License (CC BY 4.0).

http://creativecommons.org/licenses/by/4.0/

\section{(c) (i) Open Access}

\begin{abstract}
In this work, Fourier-series representation of a discontinuous function is used to highlight and clarify the controversial problem of finding the value of the function at a point of discontinuity. Several physical situations are presented to examine the consequences of this kind of representation and its impact on some widely well-known problems whose results are not clearly understood or justified.
\end{abstract}

\section{Keywords}

Electric Field, Charged Conductors, Electrostatics, Fourier Series

\section{Introduction}

The problem of a conducting sphere of radius $R$ and uniform charge $Q$ on its surface is discussed in introductory physics textbooks [1] [2]. For such a sphere, Gauss's law gives that the electric field, at a point $r$, is zero inside $(r<R)$ and $k Q / r^{2}$ outside $(r>R)$. Usually, the question of finding the electric field on points on the surface of the sphere is not raised due to the discontinuity of the field at such points. The limit of the electric field as $r \rightarrow R$ from inside gives zero and from outside gives $k Q / R^{2}$ and therefore the electric field is ill-defined on the surface. Most textbooks as in [1] and [2], authors calculate, by direct use of Gauss law, the electric field just outside a conducting surface and obtain the correct value $k Q / R^{2}$. This value is the same as the outside limiting value of the electric field of the conducting sphere. This causes a misleading and confusion among students for the electric field on the surface and may claim that the electric field there is $k Q / R^{2}$. This is not a correct conclusion and one should admit *Corresponding author. 
the ambiguity of the electric field on the surface and care must be taken when dealing with such a situation. In some cases one has to assign a value for the electric field at a very small patch on the surface for the purpose of calculating the electrostatic pressure (force per unit area) on the surface. This has been done by Griffiths in his Electrodynamics book [3]. Griffiths assigned the average value between the inside and outside limits and got the value $k Q / 2 R^{2}$ for the electric field on the surface of the conductor, and he arrived at the correct well-known value of the electrostatic pressure on the surface [4], namely, $\sigma^{2} / 2 \varepsilon_{0}$, where $\sigma$ is the surface charge density and $\varepsilon_{0}$ is the electric permittivity of free pace. Other researchers [5] [6] [7] [8] [9] considered the electric field which suffers a jump at the surface and concluded that the appropriate value of the electric field at the surface is the average value between the inside and the outside limits.

In the light of the above debate and due to the ambiguity of this problem, the aim of the present paper is to give a proof that the value of the electric field at the discontinuity point on the surface is in fact the average value between the inside and the outside limits of the electric field as $r \rightarrow R$. Fourier series expansion will be used to obtain our result.

\section{Fourier Series Representation of a Discontinuous Function}

Expansion of a function by a Fourier series has been of a great advantage in physics and engineering [10]-[18] because it allows one to more easily manipulate functions that are discontinuous or difficult to represent analytically. Some investigators have been working on fast and accurate simulations for Fourier representation of discontinuous function that represent scientific problems [19]-[25]. The value of the expanded function $f(x)$ at its point of discontinuity point is the average of the upper and lower limits of the function [26] [27]. This means that the Fourier series converges to half-way between these two limits. Mathematically, at a point of discontinuity, $x_{0}$, the Fourier series converges to the value $f\left(x_{0}\right)$ given by

$$
f\left(x_{0}\right)=\frac{1}{2} \lim _{\varepsilon \rightarrow 0}\left[f\left(x_{0}+\varepsilon\right)+f\left(x_{0}-\varepsilon\right)\right]
$$

The Fourier series expansion of the function $f(x)$, with period $2 L$ is conventionally written as

$$
f(x)=\frac{a_{0}}{2}+\sum_{n=1}^{\infty}\left[a_{n} \cos \left(\frac{n \pi x}{L}\right)+b_{n} \sin \left(\frac{n \pi x}{L}\right)\right]
$$

where $a_{0}, a_{n}, b_{n}$ are constants called Fourier coefficients. Due to the orthogonality of the sine and cosine functions, the Fourier coefficients are readily obtained with the result

$$
\begin{gathered}
a_{0}=\frac{1}{L} \int_{-L}^{L} f(x) \mathrm{d} x \\
a_{n}=\frac{1}{L} \int_{-L}^{L} f(x) \cos \left(\frac{n \pi x}{L}\right) \mathrm{d} x
\end{gathered}
$$




$$
b_{n}=\frac{1}{L} \int_{-L}^{L} f(x) \sin \left(\frac{n \pi x}{L}\right) \mathrm{d} x
$$

The factor $\frac{1}{2}$ which appears in the $a_{0}$ term in Equation (2) is included so that Equation (4) may be applied for $n=0$ as well as $n>0$.

In order to demonstrate Equation (1), we consider a specific function $f(x)$, which is given by

$$
f(x)= \begin{cases}0 & x \in(-L, 0) \\ 1 & x \in(0, L)\end{cases}
$$

Applying Equations (3)-(5), one immediately gets $a_{0}=1, a_{n}=0$ and $b_{n}=2 / n \pi$ for $n=$ odd only. Therefore, Equation (2) yields

$$
f(x)=\frac{1}{2}+\frac{2}{\pi} \sum_{n=\text { odd }}^{\infty} \frac{1}{n} \sin \left(\frac{n \pi x}{L}\right)
$$

Obviously, at the point of discontinuity $(x=0)$ the series in Equation (7) converges to $1 / 2$, which is the average value between the two limits of $f(x)$ below and above the jump.

\section{A Uniformly Charged Conducting Sphere}

In order to discuss the problem of the value of the electric field on the surface of a conducting sphere, we consider a conducting sphere of radius $R$ which is uniformly charged by a charge $Q$. Direct application of Gauss's law gives the electric field at a point $r$ from the sphere's center, with the result

$$
\vec{E}= \begin{cases}0 & r<R \\ \frac{k Q}{r^{2}} \hat{r} & r>R\end{cases}
$$

with $k=1 / 4 \pi \varepsilon_{0}$. Obviously, the electric field suffers a discontinuity at points on the surface since its limit from inside gives zero while from outside gives $k Q / R^{2}$. In order to find the electric field at points on the surface, we proceed as follows: We consider a conducting object which carries a surface charge density $\sigma$ on its surface. The widely well-known quantity which is usually invoked is the electric field just outside the conductor. The usual treatment to derive this is by considering a cylindrical Gaussian surface whose half of its length inside the conductor and the other half is outside as in Figure 2. Application of Gauss's law yields $E=\sigma / \varepsilon_{0}$, which is found in most standard introductory physics textbooks [2]. Therefore, the electric field is zero inside the conductor and a constant $\left(\sigma / \varepsilon_{0}\right)$ just outside. The result shows that the electric field suffers a finite jump on the surface, so that the electric field is written as

$$
E= \begin{cases}0 & -\varepsilon \leq x<0 \\ \frac{\sigma}{\varepsilon_{0}} & 0<x \leq \varepsilon\end{cases}
$$


where $\varepsilon$ is a small quantity and $x=0$, the discontinuity point, represents a point on the surface. The point now is to expand the electric field in Fourier series over the interval $[-\varepsilon, \varepsilon]$. Comparing Equation (9) with Equation (6) and noting that $L \rightarrow \varepsilon$, the Fourier coefficients, given in Equations (3)-(5) are easily calculated with the result $a_{0}=\sigma / \varepsilon_{0}, a_{n}=0$ and $b_{n}=2 \sigma / n \pi \varepsilon_{0}$. Therefore, Equation (2) gives the Fourier expansion of the electric field which is given by

$$
E=\frac{\sigma}{2 \varepsilon_{0}}+\frac{2 \sigma}{\pi \varepsilon_{0}} \sum_{n=\text { odd }}^{\infty} \frac{1}{n} \sin \left(\frac{n \pi x}{\varepsilon}\right)
$$

It should be clear that the above series converges to $\sigma / 2 \varepsilon_{0}$ at the discontinuity point $(x=0)$ which is the average value of the electric field between its two limits from inside and outside. Therefore, by using the relation $\sigma=q / 4 \pi R^{2}$ for the conducting sphere problem, we get our required result for the electric field on the surface,

$$
E=\frac{1}{2}\left[\lim _{r \rightarrow-R} E+\lim _{r \rightarrow+_{R}} E\right]=\frac{1}{2} \frac{Q}{4 \pi \varepsilon_{0} R^{2}}=\frac{k Q}{2 R^{2}}
$$

Our result in Equation (11) removes the ambiguity of the electric field at points on the surface of a conducting sphere and assigns a value of this field at its discontinuity point. This interesting result must be explained at the undergraduate level for physics and engineering students, since it has been avoided in almost all undergraduate physics textbooks. It should be emphasized that our result, beside its mathematical interest, it also has applications in physical situations in which the value of a function at its point of discontinuity is necessary in order to derive some relevant physical quantities. In the next section, three physical situations will be presented to demonstrate the use of our result and to show that the value of a discontinuous function at its point of discontinuity is the average value of the function at that point, which is the value where the Fourier series of the function converges.

\section{Some Physical Problems Involve Discontinuous Functions}

Here, we consider three physical situations that involve functions that suffer a jump at a point on a boundary. Therefore, assigning a value of the function at that point is necessary in order to achieve a relevant physical quantity.

\subsection{The First Problem: Electrostatic Pressure on Surface of a Conductor}

The first problem deals with the calculation of electrostatic pressure on the surface of a conductor which contains a surface charge density $\sigma$. This problem has been discussed by Griffiths [28] in his famous textbook on electrodynamics. There, Griffiths considered a patch on the surface of the conductor in attempt to find the electrostatic pressure on the surface of the conductor. He argued (but not rigorously) that the electric field on the patch is the average value between the value of electric field just outside the conductor 
$\left(\sigma / \varepsilon_{0}\right)$ and its zero value inside. Hence he arrived at the value $\sigma / 2 \varepsilon_{0}$ at the patch, and therefore the pressure (force per unit area) is just $\sigma^{2} / 2 \varepsilon_{0}$. However, this argument seems a bit dodgy because the patch is not a point so that part of the patch creates a field that affects the other part of the patch. So our result can now be applied to determine the value of the electric field at a point on the surface. For that purpose, we construct a small cylindrical surface as shown in Figure 1. We assume $\varepsilon$ to be very small, so that, $\lim _{\varepsilon \rightarrow 0^{-}} E=0$ and $\lim _{\varepsilon \rightarrow 0^{+}} E=\sigma / \varepsilon_{0}$. Therefore, the average of these two limits gives the correct value of the electric field at a point on the surface of the conducting sphere, namely, $E=\sigma / 2 \varepsilon_{0}$ and hence the well-known value of the electrostatic pressure on the surface of the sphere.

\subsection{The Second Problem: Energy Aspects in Charging a Capacitor}

The well-known two capacitor problem has been of great interest since long time ago, and variety of approaches have been considered to explain the energy loss in this problem [29] [30] [31] [32]. The essence of the problem amounts to the problem of charging a capacitor of capacitance $C$ by a power supply of electromotive force $V_{0}$ with a series resistor of resistance $R$. At the end of charging, the energy stored in the capacitor will be $\left(\frac{1}{2}\right) C V_{0}^{2}$ and exactly the same amount will be dissipated regardless of the value of $R$. In ref. [30], the authors used superconducting wires and used the flux of energy carried by the Poynting vector to calculate the energy stored in the capacitor and the energy loss. In such situation, the charge on the capacitor involves a step function, namely, $Q(t)=Q \theta(t)$. In their derivation of their final result for the stored energy, $U$, they encountered the integral

$$
U=-\frac{Q^{2}}{C} \int \theta(t) \delta(t) \mathrm{d} t=\frac{Q^{2}}{C} \theta(0)
$$

and a similar one for the energy loss. The value of the step function at the discontinuity point $(t=0)$ was used by taking the average value between the limits from below and above $t=0$ as

$$
\theta(0)=\frac{1}{2}\left[\theta\left(0^{-}\right)+\theta\left(0^{+}\right)\right]=\frac{1}{2}
$$

Therefore, the average value at the discontinuity point has a crucial role in deriving the energy loss in this process.

\subsection{The Fermi Distribution Function}

In this subsection, we present our third physical situation for the use of the average value at the jump of a discontinuous function. The average number of fermions, in a single-particle state with energy $\varepsilon$ is given by the Fermi-Dirac distribution function [33],

$$
\langle n(\varepsilon)\rangle=\frac{1}{\mathrm{e}^{(\varepsilon-\mu) / k_{B} T}+1}
$$




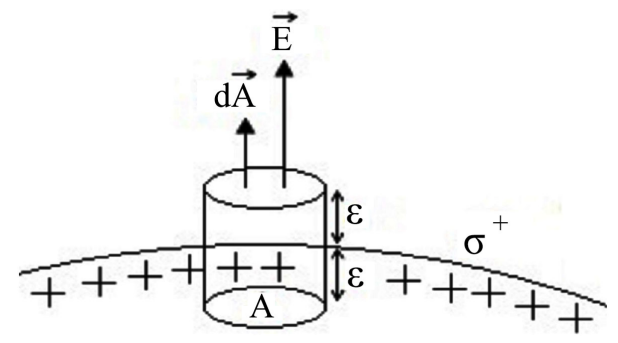

Figure 1. Cylindrical surface on the surface of a conducting sphere.

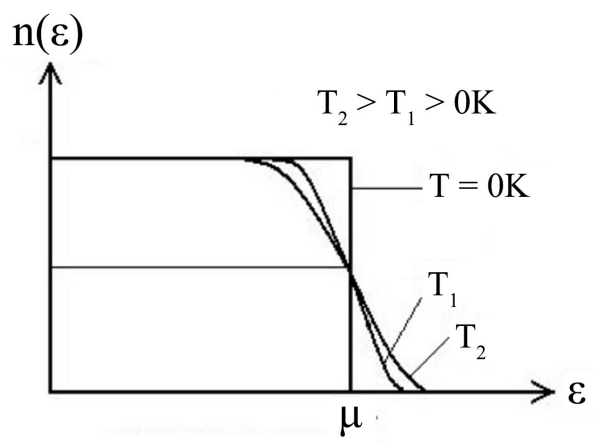

Figure 2. Fermi distribution function as function of energy at different temperatures.

where $\mu$ is the chemical potential, $k_{B}$ is the Boltzmann constant and $T$ is the temperature in Kelvin, as shown in Figure 2. We note that the Fermi distribution function behaves like a step function

$$
\lim _{T \rightarrow 0}\langle n(\varepsilon)\rangle=\left\{\begin{array}{ll}
0 & \varepsilon>\mu \\
1 & \varepsilon<\mu
\end{array}=\theta(\varepsilon-\mu)\right.
$$

Therefore, the average population number of fermions at zero temperature is $1 / 2$, which is at the midpoint of the jump. One can also observe form Equation (14) that for any temperature $T>0$, the average population number is $1 / 2$ when the energy is equal to the chemical potential.

\section{Conclusion}

In this paper, the authors examined the behavior of a discontinuous function and its application in some physical systems. Fourier series representation of such function has been studied, and it has been pointed out that, at the point of discontinuity, this series converges to the average value between the two limits of the function about the jump point. So for a step function, this convergence occurs at the exact value of one half. The obtained result clarifies and solves a controversial problem about the value of the electrostatic field at points on the surface of a conducting sphere, which is usually avoided in introductory physics books. As an application of our result, three physical systems have been discussed and the average value of the function at the discontinuity point has been used in such systems: The first deals with the calculation of the electrostatic pressure on the sur- 
face of a conductor, the second concerns the calculation of the energy loss in charging a capacitor using superconducting wires and the third is the behavior of the population function of Fermi gas at zero temperature.

\section{Conflicts of Interest}

The authors declare no conflicts of interest regarding the publication of this paper.

\section{References}

[1] Halliday, D., Resnick, R. and Walker, J. (2013) Fundamentals of Physics. 10th Edition, John Wiley \& Sons Ltd., Chichester.

[2] Serway, R.A. and Jewett, J.W. (2014) Physics for Scientists and Engineers. 9th Edition, Physics \& Astronomy, Charlie Hartford.

[3] Griffiths, D. (2017) Introduction to Electrodynamics. 4th Edition, Cambridge University Press, Cambridge.

[4] Jackson, J.D. (1999) Introduction to Electrodynamics. 3rd Edition, Wiley, New York.

[5] Gani, S. (2008) A Little Subtlety on an Electrostatic Problem. Revista Brasileira de Fesica, 30, 1701. https://doi.org/10.1590/S0102-47442008000100019

[6] Purcell, E.M. (1965) Berkeley Physics Course. Vol. 2, Mac-Graw Hill, New York.

[7] Lima, F. (2018) What Exactly Is the Electric Field at the Surface of a Charged Conducting Sphere? Resonance, 23, 1215-1223. https://doi.org/10.1007/s12045-018-0731-y

[8] Assad, G. (2012) Electric Field "On the Surface" of a Spherical Conductor: An Issue to Be Clarified. Revista Brasileira de Fesica, 34, 4701.

[9] Griffiths, D. and Walborn, S. (1999) Dirac Deltas and Discontinuous Functions. American Journal of Physics, 67, 446-447. https://doi.org/10.1119/1.19283

[10] Fedak, W.A. (2002) Quantum Jumps and Classical Harmonics. American Journal of Physics, 70, 332-344. https://doi.org/10.1119/1.1445405

[11] Fan, G.-X. (2004) Fast Fourier Transform for Discontinuous Functions. IEEE Transactions on Antennas and Propagation, 52, 461-465. https://doi.org/10.1109/TAP.2004.823965

[12] Janssen, J.M. (1950) The Method of Discontinuities in Fourier Analysis. Philips Research Reports, 5, 435-460.

[13] Huang, X., Liu, X. and Mi, Y. (2013) The Fourier Series Approach to Investigate Phase-Locking Behaviors of the Sinoatrial Node Cell. Europhysics Letters, 104, Article ID: 38002. https://doi.org/10.1209/0295-5075/104/38002

[14] Thompson, W.J. (1992) Fourier Series and Gibbs Phenomenon. American Journal of Physics, 60, 425. https://doi.org/10.1119/1.16895

[15] Tsagareishvill, V. (2017) On Fourier Coefficients of Functions with Respect to General Orthonormal Systems. Izvestiya Mathematics, 81, 179. https://doi.org/10.1070/IM8394

[16] Kvernadze, G. (2003) Approximating the Jump Discontinuities of a Function by Its Fourier-Jacobi Coefficients. Mathematics of Computation, 73, 731-751. https://doi.org/10.1090/S0025-5718-03-01594-1

[17] Ageev, A.L. and Antonova, T.V. (2015) Approximation of Discontinuity Lines for a 
Noisy Function of Two Variables with Countably Many Singularities. Journal of Applied and Industrial Mathematics, 9, 297-305. https://doi.org/10.1134/S1990478915030011

[18] Sarra, S.A. and Bai, Y. (2018) A Rational Radial Basis Function Method for Accurately Resolving Discontinuities and Steep Gradients. Applied Numerical Mathematics, 130, 131-142. https://doi.org/10.1016/j.apnum.2018.04.001

[19] Knut, S.E. (1993) Accurate and Efficient Reconstruction of Discontinuous Functions from Truncated Series Expansions. Mathematics of Computation, 61, 745-763. https://doi.org/10.1090/S0025-5718-1993-1195430-1

[20] Wright, R.K. (2002) A Roobust Method for Accurately Representing Nonperiodic Functions Given Fourier Coefficient Information. Journal of Computational and Applied Mathematics, 140, 837. https://doi.org/10.1016/S0377-0427(01)00518-0

[21] Singh, I. and Kaur, B. (2018) Teaching Graphical Simulations of Fourier Series Expansion of Some Periodic Waves Using Spreadsheets. Physics Education, 53, Article Number: 035031. https://doi.org/10.1088/1361-6552/aab447

[22] Ellerington, N., Bschaden, B., Hubbard, T. and Kujath, M. (2012) Fourier Analysis of Blurred Images for the Measurement of the In-Plane Dynamics of MEMS. Journal of Micromechanics and Microengineering, 22, Article Number: 035019. https://doi.org/10.1088/0960-1317/22/3/035019

[23] Janett, G. (2019) Discontinuities in Numerical Radiative Transfer. Astronomy \& Astrophysics, 622, A162. https://doi.org/10.1051/0004-6361/201833984

[24] Jackeman, J., Archibald, R. and Xiu, D. (2011) Characterization of Discontinuities in High Dimensional Stochastic Problems on Adaptive Sparce Grids. Journal of Computational Physics, 230, 3977-3997. https://doi.org/10.1016/j.jcp.2011.02.022

[25] Wang, X., Guo, Y., Zhang, D. and Liu, H. (2017) Fourier Method for Recovering Acoustic Sources from Multi-Frequency Far-Field Data. Inverse Problem, 33, Article Number: 035001. https://doi.org/10.1088/1361-6420/aa573c

[26] Riley, K.F., Hobson, M.P. and Bence, S.J. (2006) Mathematical Methods for Physics and Engineering. 3rd Edition, Cambridge University Press, Cambridge.

[27] Dass, H.K. and Verma, R. (2008) Mathematical Physics. Revised Edition, Chand Publishing, New York.

[28] Griffiths, D. (1999) Introduction to Electrodynamics. 4th Edition, Cambridge University Press, Cambridge.

[29] Boykin, T., Hite, D. and Singh, N. (2002) The Two Capacitor Problem with Radiation. American Journal of Physics, 71, 415-420. https://doi.org/10.1119/1.1435344

[30] AL-Jaber, S. and Salih, S. (2000) Energy Considerations in the Two-Capacitor Problem. European Journal of Physics, 21, 341-345. https://doi.org/10.1088/0143-0807/21/4/307

[31] AL-Jaber, S. and Abu-Labadeh, A. (2008) Energy Consideration of Transition from Non-Equilibrium to Equilibrium State in the Process of Charging a Capacitor. Journal of Electrostatics, 66, 190-192. https://doi.org/10.1016/j.elstat.2007.12.002

[32] Lara, V., Lima, A. and Costa, A. (2015) Entrropic Considerations in the Two-Capacitor Problem. RevistaBrasileira de Fesica, 37, 1306-1309. https://doi.org/10.1590/S1806-11173711650

[33] Pathria, R.K. and Beale, P.D. (2011) Statistical Mechanics. 3rd Edition, Academic Press, New York. 